\title{
Biosensors for Health Applications
}

\author{
Cibele Marli Cação Paiva Gouvêa \\ Universidade Federal de Alfenas \\ Brazil
}

\section{Introduction}

The ability to assess health status, disease onset and progression, and monitor treatment outcome through a non-invasive method is the main aim to be achieved in health care promotion and delivery and research. There are three prerequisites to reach this goal: specific biomarkers that indicates a healthy or diseased state; a non-invasive approach to detect and monitor the biomarkers; and the technologies to discriminate the biomarkers.

The early disease diagnosis is crucial for patient survival and successful prognosis of the disease, so that sensitive and specific methods are required for that. Among the numerous mankind diseases, three of them are relevant because of their worldwide incidence, prevalence, morbidity and mortality, namely diabetes, cardiovascular disease and cancer.

In recent years, the demand has grown in the field of medical diagnostics for simple and disposable devices that also demonstrate fast response times, are user-friendly, costefficient, and are suitable for mass production. Biosensor technologies offer the potential to fulfill these criteria through an interdisciplinary combination of approaches from nanotechnology, chemistry and medical science.

The emphasis of this chapter is on the recent advances on the biosensors for diabetes, cardiovascular disease and cancer detection and monitoring. An overview at biorecognition elements and transduction technology will be presented as well as the biomarkers and biosensing systems currently used to detect the onset and monitor the progression of the selected diseases. The last part will discuss some challenges and future directions on this field.

\section{Biorecognition elements and transduction technology}

\subsection{Biorecognition elements}

Clinical analyses are no longer carried out exclusively in the clinical chemistry laboratory. Measurements of analytes in biological fluids are routinely performed in various locations, including hospital, by caregivers in non-hospital settings and by patients at home. Biosensors (bioanalytical sensors) for the measurement of analytes of interest in clinical chemistry are ideally suited for these new applications. These factors make biosensors very attractive compared to contemporary chromatographic and spectroscopic techniques.

A biosensor can be generally defined as a device that consists of a biological recognition system and a transducer, for signal processing, to deduce and quantity a particular analyte (Hall, 1990). Biosensors provide advanced platforms for biomarker analysis with the advantages of being easy to use, rapid and robust as well as offering multianalyte testing 
capability; however a specific biomarker is necessary. Biomarkers are molecules that can be objectively measured and evaluated as indicators of normal or disease processes and pharmacologic responses to therapeutic intervention (Rusling et al., 2010).

The first biosensor was reported by Clark and Lyons (1962) for glucose in blood measurement. They coupled the enzyme glucose oxidase to an amperometric electrode for $\mathrm{PO}_{2}$. The enzyme-catalyzed oxidation of glucose consumed $\mathrm{O}_{2}$ and lowered $\mathrm{PO}_{2}$ that was sensed, proportionally to the glucose concentration in the sample. The enzyme-based sensor was the first generation of biosensors and in the subsequent years a variety of biosensors for other clinically important substances were developed. Therefore, biosensors can be categorized according to the biological recognition element (enzymatic, immuno, DNA and whole-cell biosensors; Spichiger-Keller, 1998) or the signal transduction method (electrochemical, optical, thermal and mass-based biosensors; Wanekaya et al., 2008) (Fig 1).

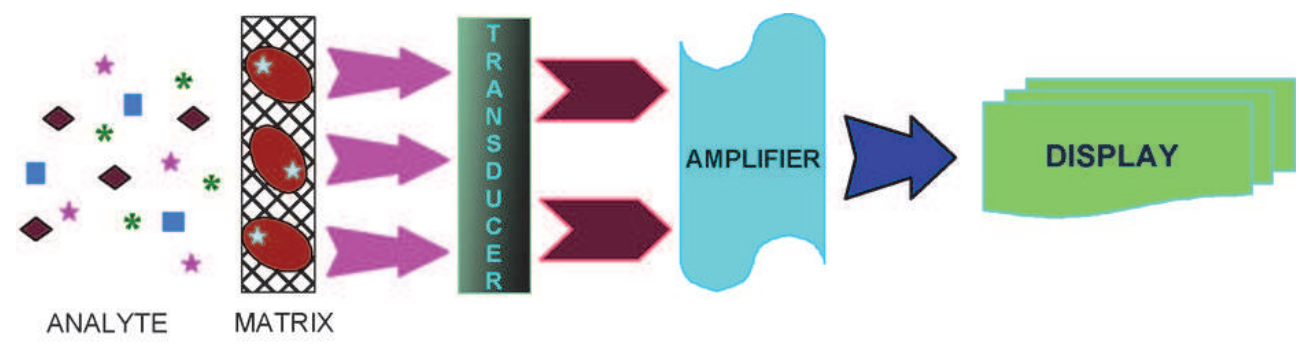

ENZYME $\rightarrow \star \star$ ACTIVE SITE

Fig. 1. Schematic of a biosensor (Arya et al., 2008).

Substantial amounts of published work on the enzyme-based biosensors are found in the literature due to their medical applicability, commercial availability or ease of enzyme isolation and purification from different sources and also enzymes can be used in combination for detection of a target analyte (D'Orazio, 2003). By acting as biocatalytic elements, the enzymatic reaction is accompanied by the consumption or production of species such as $\mathrm{CO}_{2}, \mathrm{NH}_{3}, \mathrm{H}_{2} \mathrm{O}_{2}, \mathrm{H}^{+}, \mathrm{O}_{2}$ or by the activation/inhibition activity that can be detected easily by various transducers and correlate this species to the substrates. Amongst various enzymes, glucose oxidase, horseradish peroxidase, and alkaline phosphatase have been employed in most biosensor studies (Laschi et al., 2000; Wang, 2000). The detection limit is satisfactory or exceeded but the enzyme stability is still a problem, especially considering a long period of time. A major advantage of enzyme-based biosensors is the ability, in some cases, to modify catalytic properties or substrate specificity by genetic engineering. The major limitation is the lack of specificity in differentiating among compounds of similar classes (Buerk, 1993; 2001; D'Orazio, 2003).

Affinity biosensors have received considerable attention in the last years, since they provide information about binding of antibodies to antigens, cell receptors to their ligands, DNA/RNA to complementary sequences of nucleic acids and functioning enzymatic pathways that allow the screening of gene products for metabolic functions.

Immunosensors are based on the high selectivity of the antibody-antigen reaction. The specific interaction is sensed by a transducer and measurements can be obtained directly, in minutes, rather than the hours required for visualizing results of an ELISA test 
(Spangler et al., 2001). Either an antigen or antibody can be immobilized onto a surface of support in an array format (Huang et al., 2004) and participates in a biospecific interaction with the other component, allowing detection and quantification of an analyte of interest (Stefan et al., 2000). The sensors may operate either as direct or as indirect sensors often referred to homogeneous and heterogeneous immunosensors, respectively. Antibodies are the critical part of an immunosensor to provide sensitivity and specificity. As the antibody-antigen complex is almost irreversible, only a single immunoassay can be performed (Buerk, 1993) although intensive research effort has been directed toward the regeneration of renewable antibody surfaces. Reproducibility is another concern, partly due to the antibody orientation and immobilization onto the sensor surface. Immunosensors are inherently more versatile than enzyme-based biosensors because antibodies are more selective and specific. Immunosensors are currently been used for infectious diseases diagnosis (Huang et al., 2004).

DNA analysis is the most recent and most promising application of biosensors to clinical chemistry. DNA is well suited for biosensing because the base pairing interactions between complementary sequences are both specific and robust. DNA biosensors employ immobilized relatively short synthetic single-stranded oligodeoxynucleotides that hybridizes to a complementary target DNA in the sample (Palecek, 2002). Hybridization can be performed either in solution or on solid supports. The system can be used for repeated analysis since the nucleic acid ligands can be denatured to reverse binding and then regenerated (Ivnitski et al., 1999). However, considerable research is still needed to develop methods for directly targeting natural DNA present in organisms and in human blood with high detection sensitivity (Palecek, 2002). Accurate tests for recognizing DNA sequences, usually, need to multiply small amounts of DNA into readable quantities using the polymerase chain reaction (PCR). Some of the new gene chips are sensitive enough to eliminate the need for target amplification, a time-consuming process. This improvement has stimulated the development of DNA biosensors with a view toward rapid analysis for point-of-care diagnostics for infectious disease, testing cancer and genetic disease diagnosis and measurement of drug resistance or susceptibility, and even a whole cancer circulating cell can be identified (Liu et al., 2009).

Whole-cell biosensors are based in the general metabolic status of bacteria, fungi, yeasts, animal or plant cells that are the recognition elements. Whole cells can easily be manipulated and adapted to consume and degrade new substrates. Many enzymes and cofactors that co-exist in the cells give them the ability to consume and hence detect a large number of chemicals. However, this may compromise their selectivity (Ding et al., 2008).

The sensing molecule, in general, is hold on a solid support, the matrix. Chemical properties of a desired support decide the method of immobilization and the operational stability of a biosensor. In particular, it should be resistant to a wide range of physiological pHs, temperature, ionic strength and chemical composition. The ability to co-immobilize more than one biologically active component is desirable in some cases. Conducting polymers, carbon nanotubes, nanoparticles, sol-gel/hydro-gels and self-assembled monolayer are common used to immobilize a variety of sensing molecules (Arya et al., 2008).

\subsection{Transduction technology}

The interaction of the analyte with the bioreceptor is designed to produce an effect measured by the transducer, which converts the information into a measurable signal. A variety of transducer methods have been feasible toward the development of biosensor 
technology; however the most common methods are electrochemical, optical and piezoelectric (Buerk, 1993; Collings \& Caruso 1997; Wang, 2000).

Electrochemical sensors measure the electrochemical changes that occur when analytes interact with a sensing surface of the detecting electrode. The electrochemical assay is simple, reliable, has a low detection limit and a wide dynamic range due to the fact that the electrochemical reactions occur at the electrode-solution interfaces. Based on that and cost competitiveness, more than half of the biosensors, reported in the literature, are based on electrochemical transducers (Meadows, 1996). The electrical changes can be potentiometric (a change in the measured voltage between the indicator and reference electrodes), amperometric (a change in the measured current at a given applied voltage), or conductometric (a change in the ability of the sensing material to transport charge). Amperometry is the electrochemical technique usually applied in commercially available biosensors for clinical analyses that detect redox reactions. The electrochemical platform is suited for enzyme-based and DNA/RNA sensors, field monitoring applications (e.g. handheld) and miniaturization toward the fabrication of an implantable biosensor.

Optical transducers can be used to monitor affinity reactions and have been applied to quantitate antigenic species of interest in clinical chemistry and to study the kinetics and affinity of antigen-antibody and DNA interactions. Of particular interest have been direct optical transducers based on methods such as internal reflectance spectroscopy, surface plasmon resonance and evanescent wave sensing. Light entering an optical device is directed through optical fibers or planar waveguides toward a sensing surface and reflected back out again. The reflected light is monitored, using a detector such as a photodiode, revealing information about the physical events occurring at the sensing surface. The measured optical signals often include absorbance, fluorescence, chemiluminescence, surface plasmon resonance (to probe refractive index), or changes in light reflectivity. Optical biosensors are preferable for screening a large number of samples simultaneously; however, they cannot be easily miniaturized for insertion into the bloodstream. Most optical methods of transduction require a spectrophotometer to detect signal changes.

Mass sensors can produce a signal based on the mass of chemicals that interact with the sensing film, usually a vibrating piezoelectric quartz crystal. Acoustic wave devices, made of piezoelectric materials, are the most common sensors, which bend when a voltage is applied to the crystal. Acoustic wave sensors are operated by applying an oscillating voltage at the resonant frequency of the crystal, and measuring the change in resonant frequency when the target analyte interacts with the sensing surface. Because a significant amount of nonspecific adsorption occurs in solutions, piezoelectric sensors have received their widest use in gas phase analyses. Extremely high sensitivities are possible with these devices detecting femtogram levels of drug vapors. Similarly to optical detection, piezoelectric detection requires large sophisticated instruments to monitor the signal.

Generation of heat during a reaction can be used in a calorimetric based biosensor. Changes in solution temperature caused by the reaction are measured and compared to a sensor with no reaction to determine the analyte concentration. This approach is well suited for enzyme/substrate reactions that cause changes in solution temperature but not for receptorligand reactions because there is no temperature change at steady-state and transient measurements are very difficult to make. Calorimetric microsensors have been manufactured for detection of cholesterol in blood serum based on the enzymatically produced heat of oxidation and decomposition reactions (Caygill et al., 2010). 


\section{Biosensors for diabetes applications}

\subsection{Glucose as diabetes biomarker}

About $3 \%$ of the population worldwide suffers from diabetes, a leading cause of death, and its incidence is growing fast. Diabetes is a syndrome of disordered metabolism resulting in abnormally high blood sugar levels. Diabetic individuals are at a greater heart disease, stroke, high blood pressure, blindness, kidney failure, neurological disorders risk and other health related complications without diligent monitoring blood glucose concentrations. Through patient education, regular examinations and tighter blood glucose monitoring, many of these complications can be reduced significantly (Turner \& Pickup, 1985; Lasker, 1993). Optimal management of diabetes involves patients measuring and recording their own blood glucose levels. Under normal physiological condition, the concentration of fasting plasma glucose is in the range $6.1-6.9 \mathrm{mmolL}^{-1}$, so the variation of the blood glucose level can indicate diabetes mellitus, besides other conditions. Consequently, quantitation of the glucose content is of extreme importance, as it is the main diabetes biomarker. The American Diabetes Association recommends that insulin-dependent type 1 diabetics selfmonitor blood glucose 3-4 times daily, while insulin-dependent type 2 diabetics monitor once-daily (American, 1997). However, frequent self-monitoring of glucose concentrations is difficult, given the time, the inconvenience and the discomfort involved with the traditional measurement technique. Several methods for glucose analysis have been reported. However, most of these methods involve complex procedures or are expensive in terms of costs. Therefore it is necessary to develop a simple, sensitive, accurate, micro-volume and low-cost approach for glucose analysis which is appropriate for rapid field tests and is also effective as an alternative to the existing methods.

\subsection{Biosensors for glucose measuring}

Glucose can be monitored by invasive and non-invasive technologies. Glucose biosensor was the first reported biosensor (Clark \& Lyons, 1962) and after that a great number of different glucose biosensors were developed, including implantable sensors for measuring glucose in blood or tissue. Glucose sensors are now widely available as small, minimally invasive devices that measure interstitial glucose levels in subcutaneous fat (Cengiz \& Tamborlane, 2009). Requirements of a sensor for in vivo glucose monitoring include miniaturization of the device, long-term stability, elimination of oxygen dependency, convenience to the user and biocompatibility. Long-term biocompatibility has been the main requirement and has limited the use of in vivo glucose sensors, both subcutaneously and intravascular, to short periods of time. Diffusion of low-molecular-weight substances from the sample across the polyurethane sensor outer membrane results in loss of sensor sensitivity. In order to address the problem, microdialysis or ultrafiltration technology has been coupled with glucose biosensors. The current invasive glucose monitors commercially available use glucose oxidase-based electrochemical methods and the electrochemical sensors are inserted into the interstitial fluid space. Most sensors are reasonably accurate although sensor error including drift, calibration error, and delay of the interstitial sensor value behind the blood value are still present (Castle \& Ward, 2010). The glucose biosensor is the most widely used example of an electrochemical biosensor which is based on a screenprinted amperometric disposable electrode. This type of biosensor has been used widely throughout the world for glucose testing in the home bringing diagnosis to on site analysis. 
Non-invasive glucose sensing is the ultimate goal of glucose monitoring and the main approaches being pursued for glucose sensor development are: near infrared spectroscopy, excreted physiological fluid (tears, sweat, urine, saliva) analysis, microcalorimetry, enzyme electrodes, optical sensors, sonophoresis and iontophoresis, both of which extract glucose from the skin (Koschwanez \& Reichert, 2007; Beauharnois et al., 2006; Chu et al., 2011). Despite the relative ease of use, speed and minimal risk of infection involved with infrared spectroscopy, this technique is hindered by the low sensitivity, poor selectivity, frequently required calibrations, and difficulties with miniaturization. Problems surrounding direct glucose analysis through excreted physiological fluids include a weak correlation between excreted fluids and blood glucose concentrations. Exercise and diet that alter glucose concentrations in the fluids also produce inaccurate results (Pickup et al., 2005). The desire to create an artificial pancreas drives for continued research efforts in the biosensor area. Nevertheless, the drawbacks of in vivo biosensors must be solved before such an insulin modulating system can be achieved.

\section{Biosensors for cardiovascular diseases applications}

\subsection{Cardiovascular disease biomarkers}

Cardiovascular diseases are highly preventable, yet they are major cause of death of humans over the world. One of the most important reasons of the increasing incidences of cardiovascular diseases and cardiac arrest is hypercholesterolemia, i.e. increased concentration of cholesterol in blood (Franco et al., 2011). Hence estimation of cholesterol level in blood is important in clinical applications. The early evaluation of patients with symptoms that indicates an acute coronary syndrome is of great clinical relevance. Biomarkers have become increasingly important in this setting to supplement electrocardiographic findings and patient history because one or both can be misleading. Cardiac troponin is the only marker used routinely nowadays in this setting because it is specific from the myocardial tissue, easily detected, and useful for therapeutic decision making. Determination of the level of other non-myocardial tissue-specific markers might also be helpful, such as myeloperoxidase, copeptin, growth differentiation factor 15 and Creactive protein $(\mathrm{CRP})$. CRP, which reflects different aspects of the development of atherosclerosis or acute ischemia, is one of the plasma proteins known as acute-phase proteins and its levels rise dramatically during inflammatory processes occurring in the body. This increment is due to a rise in the plasma concentration of IL-6, which is produced predominantly by macrophages as well as adipocytes. CRP can rise as high as 1000-fold with inflammation. CRP was found to be the only marker of inflammation that independently predicts the risk of a heart attack.

\subsection{Biosensors in cardiovascular disease}

Biosensors for cholesterol measurement comprise the majority of the published articles in the field of cardiovascular diseases. In the fabrication of cholesterol biosensor for the estimation of free cholesterol and total cholesterol, mainly cholesterol oxidase (ChOx) and cholesterol esterase (ChEt) have been employed as the sensing elements (Arya et al., 2008) (Fig. 2). Electrochemical transducers have been effectively utilized for the estimation of cholesterol in the system (Charpentier \& Murr, 1995; Singh et al., 2006; Zhou et al., 2006; Arya et al., 2007). Based on number and reliability of optical methods, a variety of optical transducers have been employed for cholesterol sensing, namely monitoring: luminescence, 
change in color of dye, fluorescence and others (Arya et al., 2008). Other cardiovascular disease biomarkers are also quantified. CRP measurement rely mainly on immunosensing technologies with optical, electrochemical and acoustic transducers besides approaches to simultaneous analytes measurement (Albrecht et al., 2008; Heyduk et al., 2008; McBride \& Cooper, 2008; Niotis et al., 2010; Qureshi et al., 2010a,b; Sheu et al., 2010; Zhou et al., 2010). Silva et al. (2010) incorporated streptavidin polystyrene microspheres to the electrode surface of SPEs in order to increase the analytical response of the cardiac troponin $\mathrm{T}$ and Park et al. (2009) used an assay based on virus nanoparticles for troponin I highly sensitive and selective diagnostic, a protein marker for a higher risk of acute myocardial infarction. Early and accurate diagnosis of cardiovascular disease is crucial to save many lives, especially for the patients suffering the heart attack. Accurate and fast quantification of cardiac muscle specific biomarkers in the blood enables accurate diagnosis and prognosis and timely treatment of the patients. It is apparent that increasing incidences of cardiovascular diseases and cardiac arrest in contemporary society denote the necessity of the availability of cholesterol and other biomarkers biosensors. However, only a few have been successfully launched in the market. One of the reasons lays in the optimization of critical parameters, such as enzyme stabilization, quality control and instrumentation design. The efforts directed toward the development of cardiovascular disease biosensors have resulted in the commercialization of a few cholesterol biosensors. A better comprehension of the bioreagents immobilization and technological advances in the microelectronics are likely to speed up commercialization of the much needed biosensors for cardiovascular diseases.
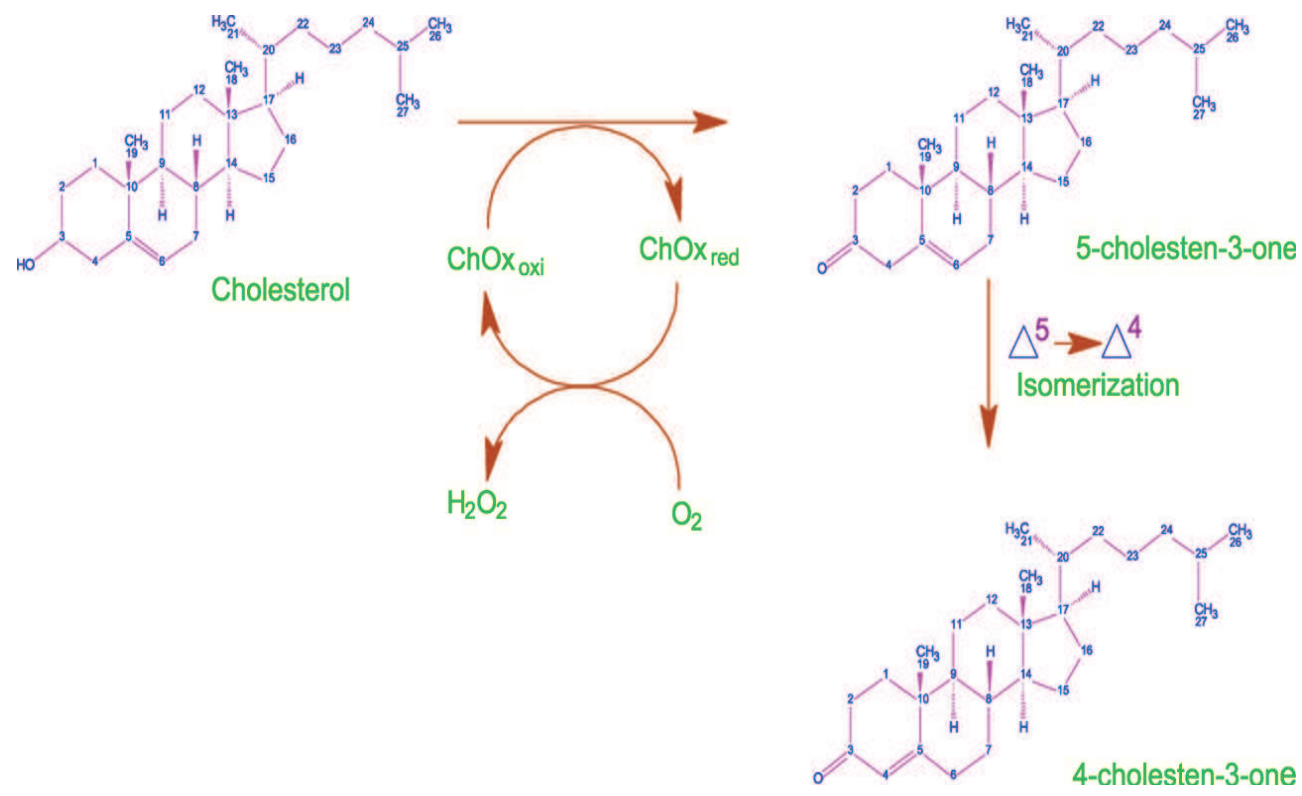

Fig. 2. Pathway of cholesterol oxidase enzyme reaction (Arya et al., 2008). 


\section{Biosensors for cancer applications}

\subsection{Cancer biomarkers}

Cancer is the leading cause of death in economically developed countries and the second leading cause of death in developing countries. This disease continues to increase globally largely because of the aging and growth of the world population alongside an increasing adoption of cancer-causing behaviors, particularly smoking. Breast cancer is the most frequently diagnosed cancer and the leading cause of cancer death among females and lung cancer is the leading cancer site in males. Breast cancer is now also the leading cause of cancer death among females in economically developing countries, a shift from the previous decade during which the most common cause of cancer death was cervical cancer (Jemal et al., 2011). Solid cancers are a leading cause of morbidity and mortality worldwide, primarily due to the failure of effective clinical detection and treatment of metastatic disease in distant sites (Chambers et al., 2002; Pantel \& Brakenhoff, 2004). Cancer can be caused by a range of factors, both genetic and environmental. Chemical, physical and biological factors such as the exposure to carcinogenic chemicals, radiation, bacterial (e.g. stomach cancer), viral infections (e.g. cervical cancer) and toxins (aflatoxin; e.g. liver cancer) can lead to cancer development (Vineis et al., 2010). As the causes of cancer are so diverse, clinical testing is also very complex. The multi-factorial changes (genetic and epigenetic) can cause the onset of the disease and the formation of cancer cells. However, no single gene is universally altered during this process, but a set of them that brings difficulties to the correct disease diagnosis. All the changes which take place, in the tumors from different locations (organ), as well within tumors from the same location, can be so variable and overlapping that it is difficult to select a specific change or marker for the diagnosis of specific cancers. Therefore, a range of biomarkers can potentially be analyzed for disease diagnosis. These biomarkers or molecular signatures can be produced either by the tumor itself or by the body in response to the presence of cancer (Robert, 2010). Several cancer biomarkers are listed in Table 1.

The analysis of biomarkers in body fluids such as blood, urine and others is one of the methods applied in the detection of the disease. Multi-marker profiles, both presence and concentration level, can be essential for the diagnosis of early disease onset. These methods should provide information to assist clinicians in making successful treatment decisions and increasing patient survival rate (Tothill, 2009). A range of biomarkers have been identified with different types of cancers. These include DNA modifications, RNA, proteins (enzymes and glycoproteins), hormones and related molecules, molecules of the immune system, oncogenes and other modified molecules. Several biomarkers are current being studied, including genes and proteins; however few of them have routine cancer clinical testing importance because of their complexity. The development of protein based biomarkers for biosensors use in cancer diagnosis is more attractive than genetic markers due to protein abundance, recovery and cost effective technique for the development of point-of-care devices (Li et al., 2010).

\subsection{Biosensors in cancer disease}

Existing methods of screening for cancer are heavily based on cell morphology using staining and microscopy which are invasive techniques. Furthermore, tissue removal can miss cancer cells at the early onset of the disease. Biosensor-based detection becomes practical and advantageous for cancer clinical testing, since it is faster, more user-friendly, 
less expensive and less technically demanding than microarray or proteomic analyses. However, significant technical development is still needed, particularly for protein based biosensors. For cancer diagnosis multi-array sensors would be beneficial for multi-marker analysis. A range of molecular recognition molecules have been used for biomarker detection, being antibodies the most widely used. More recently, synthetic (artificial) molecular recognition elements such as nanomaterials, aptamers, phage display peptides, binding proteins and synthetic peptides as well as metal oxides materials have been fabricated as affinity materials and used for analyte detection and analysis (Sadik et al., 2009; Khati, 2010). Antibodies (monoclonal and polyclonal) have been applied in cancer diagnostics tests targeting cancer cells and biomarkers. Polyclonal antibodies can be raised against any biomarker or cells and with the introduction of high throughput techniques, applying these molecules in sensors has been successful. The use of monoclonal antibodies however, results in more specific tests. The drawbacks include that monoclonal antibodies are more difficult to maintain and can be more expensive than polyclonal antibodies (Huang et al., 2010). Replacing natural biomolecules with artificial receptors or biomimics has therefore become an attractive area of research in recent years. The advantages of using these molecules are that they are robust, more stable, less expensive to produce and can be modified easily to aid immobilization on the sensor surface as well as adding labels as the maker for detection (Liu et al., 2007). Those molecules can be synthesized after a selection from combinatorial libraries with higher specificity and sensitivity when compared to the antibody molecule.

\begin{tabular}{|c|c|}
\hline Breast & $\begin{array}{c}\text { ER,PR, HER2, CA15-3, CA125, CA27.29, CEA } \\
\text { BRCA1, BRCA2, MUC-1, CEA, NY-BR-1, ING- } \\
1\end{array}$ \\
\hline Bladder & $\begin{array}{c}\text { BAT, FDP, NMP22, HA-Hase, BLCA-4, CYFRA } \\
21-1\end{array}$ \\
\hline Cervix & $\begin{array}{c}\text { P53, Bcl-2, Brn-3a, MCM, SCC-Ag, TPA, } \\
\text { CYFRA 21-1, VEGF, M-CSF }\end{array}$ \\
\hline Colon & HNPCC, FAP, CEA, CA19-9, CA24-2, p53 \\
\hline Esophagus & ChCC \\
\hline Leukemia & AFP, CEA \\
\hline Liver & NSE \\
\hline Lung & $\begin{array}{c}\text { NY-ESO-1, CEA, CA19-9, SCC, CYFRA21-1, } \\
\text { Tyrosinase, NY-ESO-1 }\end{array}$ \\
\hline Melanoma & CA125, AFP, hCG, p53, CEA \\
\hline Ovarian & CA19-9, CEA, MIC-1 \\
\hline Pancreas & PSA, PAP \\
\hline Prostate & CA72-4, CEA, CA19-9 \\
\hline Solid tumors & $\begin{array}{c}\text { Circulating tumour cells in biological fluids, } \\
\text { expression of targeted growth factor receptors }\end{array}$ \\
\hline Stomach &
\end{tabular}

Table 1. Cancer biomaker 
For cancer biomarkers analysis, bioaffinity based electrochemical biosensors are usually applied to detect gene mutations of biomarkers and protein biomarkers. Electrochemical affinity sensors based on antibodies offer great selectivity and sensitivity for early cancer diagnosis and these include amperometric, potentiometric and impedimetric/conductivity devices. Amperometric and potentiometric transducers have been the most commonly used, but much attention in recent years has been devoted to impedance based transducers since they are classified as label-free detection sensors. However, much of the technology is still at the research stage (Lin \& Ju, 2005; Wang, 2006). Besides based on antibodies, electrochemical devices have been developed based on DNA hybridization and used for cancer gene mutation detection. In this type of device a single stranded DNA sequence is immobilized on the electrode surface where DNA hybridization takes place (Ahmed, 2008). ELISA based assays conducted on the electrode surface are the most frequently used techniques for cancer protein markers analysis, such as CEA. In this method the antibody (or antigen) is labeled with an enzyme such as horseradish peroxidase (HRP), or alkaline phosphatase (AP) and these will then catalyze an added substrate to produce an electroactive species which can then be detected on an electrochemical transducer. Electrochemical detection of rare circulating tumor cells has the potential to provide clinicians with a standalone system to detect and monitor changes in cell numbers throughout therapy, conveniently and frequently for efficient cancer treatment (Chung et al., 2011).

Many commercially available platforms use fluorescence labels as the detection system. However, the instruments used for signal readout are usually expensive and are more suitable for laboratory settings. As an example the Affymetrix gene chip (Affymetrix Inc., Santa Clara, USA) can be used for screening cancer and cancer gene identification. Other biosensor platforms such as grating couplers, resonant mirrors and surface plasmon based systems have also been used for cancer biomarkers diagnosis. These are classified as labelfree and real-time affinity reaction detection systems. Different SPR based biosensors have been developed for cancer markers detection based on the above optical systems (Tothill, 2009). Recently, microcantilever based sensors have also been applied for early-stage diagnosis of hepatocellular carcinoma (Liu et al., 2009b).

In spite of the achieved development in cancer biosensing, the point-of-care testing is not yet available. In order to achieve this goal challenges must be overcome such as: development of reproducible biomarker assays; improvement in recognition ligands; development of multi-channel biosensors; advances in sample preparation; device miniaturization and integration; development of more sensitive transducers; microfluidics integration; advanced manufacturing techniques and cost reduction (Rasooly \& Jacobson, 2006).

\section{Conclusion}

A precise diagnostic for a disease is essential for a successful treatment and recovery of patients suffering from it. Diagnostics methods must be simple, sensitive and able to detect multiple biomarkers that exist at low concentrations in biological fluids. Biosensors can fulfill these requirements. However, biosensor devices need to be further developed and improved to face these new challenges to allow, for example, multiplex analysis of several biomarkers where arrays of sensors need to be developed on the same chip.

Biosensors are firmly established for application in clinical chemical analysis. Biosensors for measurement of blood metabolites such as glucose, lactate, urea and creatinine, using both electrochemical and optical modes of transduction, are commercially developed and used 
routinely in the laboratory, in point-of-care settings and, in the case of glucose, for selftesting. While immunosensors have difficulty competing with traditional immunoassay based mainly on sensitivity requirements, they hold promise for testing where some sensitivity can be sacrificed for improved ease of use and faster time to result, such as in near-patient testing for cardiac and cancer markers. Although biosensors are used for several clinical applications, few biosensors have been developed for cardiovascular and cancer-related clinical testing. Development of molecular tools, both genomic and proteomic, to profile tumors and produce molecular signatures, based on genetic and epigenetic signatures, changes in gene expression and protein profiles and protein posttranslational modifications has opened new opportunities for utilizing biosensors in cancer testing. Harnessing the potential of biosensors is challenging because of cancer's complexity and diversity. Successful development of biosensor-based cancer testing will require continued development and validation of biomarkers and development of ligands for those biomarkers, as well as continued development of sample preparation methods and multi-channel biosensors able to analyze many cancer markers simultaneously. The use of biosensors for cancer clinical testing may increase assay speed and flexibility, enable multitarget analyses and automation and reduced costs of diagnostic testing. Biosensors have the potential to deliver molecular testing to the community health care setting and to underserved populations. Cancer biomarkers identified from basic and clinical research, and from genomic and proteomic analyses must be validated. Ligands and probes for these markers can then be combined with detectors to produce biosensors for cancer-related clinical testing. Point-of-care cancer testing requires integration and automation of the technology as well as development of appropriate sample preparation methods (Rasooly \& Jacobson, 2006).

A clear direction for future work in biosensor research is in molecular diagnostics. Improving the sensitivity of DNA biosensors for a single-molecule detection in an unamplified sample is an important goal to achieve. This goal will require enhancing the signal-to-noise rate, improving the signal produced by the biochemical reaction or increasing the sensitivity of the transducer while reducing background noise. Ultrasensitive transducer technologies will be required. Some recent examples of transduction modes with enhanced sensitivity include microcantilevers for the detection of mass changes upon detection of a binding event and quartz crystal microbalances capable of monitoring formation and rupturing of chemical bonds by sensing acoustic emissions. The latter has demonstrated sensitivity to detect a single virus particle. Increasing the arrays amplitude for more complete and rapid DNA sequencing information is another area of focus, and improvements in this area may ultimately be limited by resolution of the detection transducer. DNA chips are being incorporated into total analysis systems, including microfluidics and the biosensor on a single structure. These systems should include, in the future, no need for sample preparation, a user-friendly handling system, chemical analysis and signal acquisition capabilities. Central to development of lab-on-a-chip analysis system will be the homogeneous sensing formats and microfabrication technologies for DNA analysis. One recent step towards a homogeneous assay has been the development of synthetic polymeric probes that emit fluorescence only after the hybridization to native DNA targets, allowing monitoring of hybridization in real time without the need for separation steps. Further development and improvement of nanotechnologies will be needed to produce nanoscale devices, with expanded sizes of arrays using reduced sample volume. The future of such devices for rapid determination of a disease could be especially 
used for point-of-care application. However, cost and quality control of these devices must be strictly adjusted for the accurate devices to gain popular acceptance. Homogeneous assay formats, removing the need for sample preparation and amplification steps and mass fabrication will be important to lowering cost.

Molecular biology will play a central role in the future of biosensor development, for example, to improve biocomponent stability, and for the development of aptamers. The highly reproducible synthetic approach and ease of immobilization of aptamers hold great promise for the custom design of future biosensors for molecular diagnostics (D'Orazio, 2003). Future innovation in biosensor technology to include biomarkers patterns, software and microfluidics can make these devices of high potential for health applications. The concept of using nanomaterials in the development of sensors for biomarkers diagnosis will make these devices highly sensitive and more applicable for point-of-care early diagnosis. Early diagnosis will aid in the increase in the survival rate of patients and successful development of biosensors for disease diagnosis and monitoring will require appropriate funding to move the technology from research through to the realization of commercial products.

Biosensor research and development over the past decades have demonstrated that it is still a relatively young technology. The rationale behind the slow and limited technology transfer could be attributed to cost considerations and some key technical barriers. Many of the more recent major advances had to await miniaturization technologies that are just becoming available through research in the electronic and optical solid state circuit industries. Analytical chemistry has changed considerably, driven by automation, miniaturization, and system integration with high throughput for multiple tasks. Such requirements pose a great challenge in biosensor technology which is often designed to detect one single or a few target analytes. Successful biosensors must be versatile to support interchangeable biorecognition elements, and in addition miniaturization must be feasible to allow automation for parallel sensing with ease of operation at a competitive cost. The future is very bright for biosensors. These advancements will, however, require a concerted multi-disciplinary approach for the sensor systems to successfully make the very big jump from the research and development laboratory to the market place. Combination of several new techniques, derived from physical chemistry, molecular biology, biochemistry, thick and thin film physics, materials science and electronics with the necessary expertise has revealed the promise for development of viable clinical useful biosensor.

\section{References}

Ahmed, FE. (2008). Mining the oncoproteome and studying molecular interactions for biomarker development by 2DE, ChIP and SPR technologies. Expert Review of Proteomics, Vol.5, No.3, pp. 469-496.

Albrecht, C.; Kaeppel, N. \& Gauglitz, G. (2008). Two immunoassay formats for fully automated CRP detection in human serum. Analytical and Bioanalytical Chemistry, Vol.391, No.5, pp. 1845-1852.

American diabetes association. (1997). Clinical practice recommendations 1997 Introduction. Diabetes Care, Vol.20, No. Suppl 1, pp. S1-S70.

Arya, SK.; Datta, M. \& Malhotra, BD. (2008). Recent advances in cholesterol biosensor. Biosensors and Bioelectronics, Vol.23, No.7, pp. 1083-1100. 
Arya, SK.; Prusty, AK.; Singh, SP.; Solanki, PR.; Pandey, MK.; Datta, M. \& Malhotra, BD. (2007). Cholesterol biosensor based on N-(2-aminoethyl)-3-aminopropyltrimethoxysilane self-assembled monolayer. Analytical Biochemistry, Vol.363, No.2, pp. 210-218.

Beauharnois, ME.; Neelamegham, S. \& Matta, KL. (2006). Quantitative measurement of selectin-ligand interactions: assays to identify a sweet pill in a library of carbohydrates. Methods in Molecular Biology, Vol.347, pp. 343-358.

Buerk, DG. (1993). Biosensors: Theory and Applications. Technomic Publishing Company, ISBN 0-87762-975-7, Lancaster, UK.

Castle, JR. \& Ward, WK. (2010 ). Amperometric glucose sensors: sources of error and potential benefit of redundancy. Journal of Diabetes Science and Technology, Vol.4, No. 1, pp. 221-225.

Caygill, RL.; Blair, GE. \& Millner, PA. (2010). A review on viral biosensors to detect human pathogens. Analytica Chimica Acta, Vol.681, No. 12, pp. 8-15.

Cengiz, E. \& Tamborlane, WV. (2009). A tale of two compartments: interstitial versus blood glucose monitoring. Diabetes Technology and Therapeutics, Vol.11, No. Suppl 1, pp. S11-S16.

Chambers, AF.; Groom, AC. \& MacDonald, IC. (2002). Dissemination and growth of cancer cells in metastatic sites. Nature Reviews Cancer, Vol.2, No. 8, pp. 563-572.

Charpentier, L. \& Murr, NE. (1995). Amperometric determination of cholesterol in serum with use of a renewable surface peroxidase electrode. Analytica Chimica Acta, Vol.318, No1, pp. 89-93.

Chu, MX.; Miyajima, K.; Takahashi, D.; Arakawa, T.; Sano, K.; Sawada, S.; Kudo, H.; Iwasaki, Y.; Akiyoshi, K.; Mochizuki, M. \& Mitsubayashi, K. (2011). Soft contact lens biosensor for in situ monitoring of tear glucose as non-invasive blood sugar assessment. Talanta, Vol.83, No.3, pp. 960-965.

Chung, YK.; Reboud, J.; Lee, KC.; Lim, HM.; Lim, PY.; Wang, KY.; Tang, KC.; Ji, H. \& Chen, Y. (2011). An electrical biosensor for the detection of circulating tumor cells. Biosensors and Bioelectronics, Vol.26, No. 5, pp. 2520-2526.

Clark Jr., LC. \& Lyons, C. (1962). Electrode systems for continuous monitoring in cardiovascular surgery. Annals of the New York Academy of Sciences, Vol.102, No.1, pp. 29-45.

Collings, AF. \& Caruso, F. (1997). Biosensors: recent advances. Reports on Progress in Physics, Vol.60, No.11, 1397-1445.

Ding, L.; Du, D.; Zhang, X. \& Ju, H. (2008). Trends in cell-based electrochemical biosensors. Current Medicinal Chemistry, Vol.15, No.30, pp. 3160-3170.

D'Orazio, P. (2003). Biosensors in clinical chemistry. Clinica Chimica Acta, Vol.334, No. 1-2, pp. 41-69.

Franco, M.; Cooper, RS.; Bilal, U. \& Fuster, V. (2011). Challenges and opportunities for cardiovascular disease prevention. American Journal of Medicine, Vol.24, No.2, pp. 95-102.

Hall, EAH. (1990). Biosensors. Open University Press, ISBN-10: 0335151612, Cambridge, UK.

Heyduk, E.; Dummit, B.; Chang, YH. \& Heyduk, T. (2008). Molecular pincers: antibodybased homogeneous protein sensors. Analytical Chemistry, Vol.80, No. (13):5152-9.

Huang, L.; Muyldermans, S. \& Saerens, D. (2010). Nanobodies®: proficient tools in diagnostics. Expert Review of Molecular Diagnostics, Vol.10, No. 6, pp. 777-785. 
Huang, R.; Lin, Y.; Shi, Q.; Flowers, L.; Ramachandran, S.; Horowitz, IR.; et al. (2004). Enhanced protein profiling arrays with ELISA-based amplification for highthroughput molecular changes of tumor patients' plasma. Clinical Cancer Research, Vol.10, No.2, pp. 598-609.

Ivnitski, D.; Abdel-Hamid, I.; Atanasov, P. \& Wilkins, E. (1999). Biosensors for detection of pathogenic bacteria. Biosensors and Bioelectronics, Vol.14, No.7, pp. 599-624.

Jemal, A.; Bray, F.; Center, MM.; Ferlay, J.; Ward, E. \& Forman, D. (2011). Global cancer statistics. CA: A Cancer Journal for Clinicians, Vol. 61, doi:10.3322/caac.20107.

Khati, M. The future of aptamers in medicine. (2010 ). Journal of Clinical Pathology, Vol.63, No.6, pp. 480-487.

Koschwanez, HE. \& Reichert, WM. (2007). In vitro, in vivo and post explantation testing of glucose-detecting biosensors: current methods and recommendations. Biomaterials, Vol.28, No. 25, pp. 3687-3703.

Laschi, S.; Franek, M. \& Mascini, M. (2000). Screen-printed electrochemical immunosensors for PCB detection. Electroanalysis, Vol.12, No.16, pp. 1293-1298.

Lasker, RD. (1993). The Diabetes Control and Complications Trial - Implications for Policy and Practice. New England Journal of Medicine, Vol.329, No. 14, pp. 1035-1036.

Li, Z.; Wang, Y.; Wang, J.; Tang, Z.; Pounds, JG. \& Lin, Y. (2010 ). Rapid and sensitive detection of protein biomarker using a portable fluorescence biosensor based on quantum dots and a lateral flow test strip. Analytical Chemistry, Vol.15;82, No.16, pp. 7008-7014.

Lin, J. \& Ju, H. (2005). Electrochemical and chemiluminescent immunosensors for tumor markers. Biosensors and Bioelectronics, Vol.20, No.8, pp. 1461-1470.

Liu, G.; Mao, X.; Phillips, JA.; Xu, H.; Tan, W.\& Zeng, L. (2009a). Aptamer-nanoparticle strip biosensor for sensitive detection of cancer cells. Analytical Chemistry, Vol.81, No. 24, pp. 10013-10018.

Liu, GL.; Rosa-Bauza, YT.; Salisbury, CM.; Craik, C.; Ellman, JA.; Chen, FF. \& Lee, LP. (2007). Peptide-nanoparticle hybrid SERS probes for optical detection of protease activity. Journal of Nanoscience and Nanotechnology, Vol.7, No.7, pp. 2323-2330.

Liu, Y.; Li, X.; Zhang, Z.; Zuo, G.; Cheng, Z. \& Yu, H. (2009b). Nanogram per milliliter-level immunologic detection of alpha-fetoprotein with integrated rotating-resonance microcantilevers for early-stage diagnosis of heptocellular carcinoma. Biomedical Microdevices, Vol.11, No.1, pp. 183-191.

McBride, JD. \& Cooper, MA. (2008). A high sensitivity assay for the inflammatory marker CReactive protein employing acoustic biosensing. Journal of Nanobiotechnology, Vol.6, pp. 5, 6:5doi:10.1186/1477-3155-6-5.

Meadows, D. (1996). Recent developments with biosensing technology and applications in the pharmaceutical industry. Advanced Drug Delivery Reviews, Vol.21, No.3, pp. 179189.

Niotis, AE.; Mastichiadis, C.; Petrou, PS.; Christofidis, I.; Kakabakos, SE.; Siafaka-Kapadai, A.; \& Misiakos, K. (2010). Dual-cardiac marker capillary waveguide fluoroimmunosensor based on tyramide signal amplification. Analytical and Bioanalytical Chemistry, Vol.396, No. 3, pp. 1187-1196.

Palecek, E. (2002). Past, present and future of nucleic acids electrochemistry. Talanta, Vol. 56, No.5, pp. 809-819. 
Pantel, K. \& Brakenhoff, RH. (2004). Dissecting the metastatic cascade. Nature Reviews Cancer, Vol.4, No. 6, pp. 448-456.

Park, JS.; Cho, MK.; Lee, EJ.; Ahn, KY.; Lee, KE.; Jung, JH.; Cho, Y.; Han, SS.; Kim, YK. \& Lee, J. (2009). A highly sensitive and selective diagnostic assay based on virus nanoparticles. Nature Nanotechnology, Vol.4, No. 4, pp. 259-264.

Pickup, JC.; Hussain, F.; Evans, ND. \& Sachedina, N. (2005). In vivo glucose monitoring: the clinical reality and the promise. Biosensors and Bioelectronics, Vol.20, No. 10, pp. 1897-902.

Qureshi, A.; Gurbuz, Y.; Kallempudi, S. \& Niazi, JH. (2010a). Label-free RNA aptamer-based capacitive biosensor for the detection of C-reactive protein. Physical Chemistry Chemical Physics, Vol.12, No.32, pp. 9176-982.

Qureshi, A.; Niazi, JH.; Kallempudi, S. \& Gurbuz, Y. (2010b). Label-free capacitive biosensor for sensitive detection of multiple biomarkers using gold interdigitated capacitor arrays. Biosensors and Bioelectronics, Vol.25, No. 10, pp. 2318-2323.

Rasooly, A, Jacobson J. (2006). Development of biosensors for cancer clinical testing. Biosensors and Bioelectronics, Vol.21, No. 10, pp. 1851-1858.

Robert, J. (2010). Polymorphismes génétiques. Bulletin du Cancer, Vol.97, No. 11, pp. 12531264.

Rusling, JF.; Kumar, CV.; Gutkind, JS. \& Patel, V. (2010). Measurement of biomarker proteins for point-of-care early detection and monitoring of cancer. Analyst, Vol.135, No. 10, pp. 2496-2511.

Sadik, OA.; Aluoch, AO. \& Zhou, A. (2009). Status of biomolecular recognition using electrochemical techniques. Biosensors and Bioelectronics, 24, No.9, pp. 2749-2765.

Sheu, BC.; Lin, YH.; Lin, CC.; Lee, AS.; Chang, WC.; Wu, JH.; Tsai, JC. \& Lin, S. (2010). Significance of the $\mathrm{pH}$-induced conformational changes in the structure of Creactive protein measured by dual polarization interferometry. Biosensors and Bioelectronics, Vol.26, No. 2, pp. 822-827.

Silva, BV.; Cavalcanti, IT.; Mattos, AB.; Moura, P.; Sotomayor, MP. \& Dutra, RF. (2010 ). Disposable immunosensor for human cardiac troponin $\mathrm{T}$ based on streptavidinmicrosphere modified screen-printed electrode. Biosensors and Bioelectronics, Vol.26, No. 3, pp. 1062-1067.

Singh, S.; Solanki, PR.; Pandey, MK. \& Malhotra, BD. (2006). Covalent immobilization of cholesterol esterase and cholesterol oxidase on polyaniline films for application to cholesterol biosensor. Analytica Chimica Acta, Vol.568, No.1-2, pp. 126-132.

Spangler, BD.; Wilkinson, EA.; Murphy, JT. \& Tyler, BJ. (2001). Comparison of the Spreeta ${ }^{\circledR}$ surface plasmon resonance sensor and a quartz crystal microbalance for detection of Escherichia coli heat-labile enterotoxin. Analytica Chimica Acta, Vol.444, No.1, pp. 149-161.

Spichiger-Keller, UE. (1998). Chemical sensors and biosensors for medical and biological applications. Weinheim: Wiley-VCH, ISBN 978-352-7612-28-4, Verlag, GmbH.

Stefan, RI.; van Staden, JF. \& Aboul-Enein, HY. (2000). Immunosensors in clinical analysis. Fresenius' Journal of Analytical Chemistry, Vol.366, No.6-7, pp. 659-668.

Tothill, IE. (2009). Biosensors for cancer markers diagnosis. Seminars in Cell and Developmental Biology, Vol.20, No.1, pp. 55-62.

Turner, APF. \& Pickup, JC. (1985). Diabetes-mellitus - biosensors for research and management. Biosensors, Vol.1, No. 1, pp. 85-115. 
Vineis, P.; Schatzkin, A. \& Potter, JD. (2010). Models of carcinogenesis: an overview. Carcinogenesis, Vol.31, No. 10, pp. 1703-1709

Wanekaya, AK., Chen, W. \& Mulchandani, A. (2008). Recent biosensing developments in environmental security. Journal of Environmental Monitoring, Vol. 10, No. 6, pp. 703712.

Wang, J. (2000). Analytical Electrochemistry. 2nd ed. Wiley-VCH, ISBN 0471-28272-3, New York, USA.

Wang, J. (2006). Electrochemical biosensors: towards point of care cancer diagnostics. Biosensors and Bioelectronics, Vol.21, No.10, pp. 1887-1892.

Zhou, F.; Lu, M.; Wang, W.; Bian, ZP.; Zhang, JR. \& Zhu, JJ. (2010). Electrochemical immunosensor for simultaneous detection of dual cardiac markers based on a poly(dimethylsiloxane)-gold nanoparticles composite microfluidic chip: a proof of principle. Clinical Chemistry, Vol. 56, No. 11. pp. 1701-1707.

Zhou, N.; Wang, J.; Chen, T.; Yu, Z. \& Li, G. (2006.) Enlargement of gold nanoparticles on the surface of a self-assembled monolayer modified electrode: a mode in biosensor design. Analytical Chemistry, Vol.78, No. 14, pp. 5227-5230. 


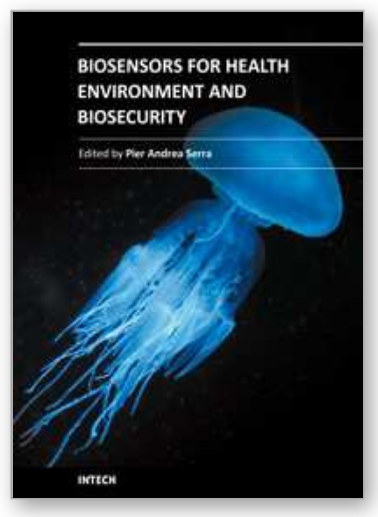

\author{
Biosensors for Health, Environment and Biosecurity \\ Edited by Prof. Pier Andrea Serra
}

ISBN 978-953-307-443-6

Hard cover, 540 pages

Publisher InTech

Published online 19, July, 2011

Published in print edition July, 2011

A biosensor is a detecting device that combines a transducer with a biologically sensitive and selective component. Biosensors can measure compounds present in the environment, chemical processes, food and human body at low cost if compared with traditional analytical techniques. This book covers a wide range of aspects and issues related to biosensor technology, bringing together researchers from 16 different countries. The book consists of 24 chapters written by 76 authors and divided in three sections: Biosensors Technology and Materials, Biosensors for Health and Biosensors for Environment and Biosecurity.

\title{
How to reference
}

In order to correctly reference this scholarly work, feel free to copy and paste the following:

Cibele Gouvea (2011). Biosensors for health applications, Biosensors for Health, Environment and Biosecurity, Prof. Pier Andrea Serra (Ed.), ISBN: 978-953-307-443-6, InTech, Available from:

http://www.intechopen.com/books/biosensors-for-health-environment-and-biosecurity/biosensors-for-healthapplications

\section{INTECH}

open science | open minds

\section{InTech Europe}

University Campus STeP Ri

Slavka Krautzeka 83/A

51000 Rijeka, Croatia

Phone: +385 (51) 770447

Fax: +385 (51) 686166

www.intechopen.com

\section{InTech China}

Unit 405, Office Block, Hotel Equatorial Shanghai

No.65, Yan An Road (West), Shanghai, 200040, China

中国上海市延安西路65号上海国际贵都大饭店办公楼 405 单元

Phone: +86-21-62489820

Fax: +86-21-62489821 
(C) 2011 The Author(s). Licensee IntechOpen. This chapter is distributed under the terms of the Creative Commons Attribution-NonCommercialShareAlike-3.0 License, which permits use, distribution and reproduction for non-commercial purposes, provided the original is properly cited and derivative works building on this content are distributed under the same license. 